\title{
A logistic analysis prediction model of TMJ condylar erosion in patients with TMJ arthralgia
}

\author{
Rüdiger Emshoff ${ }^{*}$, Annika Bertram², Linus Hupp ${ }^{3}$ and Ansgar Rudisch ${ }^{4}$
}

\begin{abstract}
Background: In terms of diagnostic and therapeutic management, clinicians should adequately address the frequent aspects of temporomandibular joint (TMJ) osteoarthritis (OA) associated with disk displacement. Condylar erosion (CE) is considered an inflammatory subset of OA and is regarded as a sign of progressive OA changes potentially contributing to changes in dentofacial morphology or limited mandibular growth. The purpose of this study was to establish a risk prediction model of CE by a multivariate logistic regression analysis to predict the individual risk of CE in TMJ arthralgia. It was hypothesized that there was a closer association between CE and magnetic resonance imaging (MRI) indicators.
\end{abstract}

Methods: This retrospective paired-design study enrolled 124 consecutive TMJ pain patients and analyzed the clinical and TMJ-related MRI data in predicting CE. TMJ pain patients were categorized according to the research diagnostic criteria for temporomandibular disorders (RDC/TMD) Axis I protocol. Each patient underwent MRI examination of both TMJs, 1-7 days following clinical examination.

Results: In the univariate analysis analyses, 9 influencing factors were related to CE, of which the following 4 as predictors determined the binary multivariate logistic regression model: missing posterior teeth (odds ratio $[\mathrm{OR}]=1.42 ; P=0.018), \mathrm{RDC} / \mathrm{TMD}$ of arthralgia coexistant with disk displacement without reduction with limited opening (DDwoR/wLO) $(\mathrm{OR}=3.30, P=0.007)$, MRI finding of disk displacement without reduction $(\mathrm{OR}=10.96$, $P<0.001)$, and MRI finding of bone marrow edema $(O R=11.97, P<0.001)$. The model had statistical significance (chi-square $=148.239$, Nagelkerke $R$ square $=0.612, P<0.001$ ). Out of the TMJs, $83.9 \%$ were correctly predicted to be CE cases or Non-CE cases with a sensitivity of $81.4 \%$ and a specificity of $85.2 \%$. The area under the receiver operating characteristic curve was 0.916.

Conclusion: The established prediction model using the risk factors of TMJ arthralgia may be useful for predicting the risk of CE. The data suggest MRI indicators as dominant factors in the definition of CE. Further research is needed to improve the model, and confirm the validity and reliability of the model.

Keywords: Temporomandibular joint, Arthralgia, Magnetic resonance imaging, Disk displacement, Condylar erosion, Bone marrow edema, Predictive modeling

\footnotetext{
*Correspondence: ruediger.emshoff@tirol-kliniken.at

${ }^{1}$ Orofacial Pain and TMD Unit, University Clinic of Oral and Maxillofacial Surgery, Medical University of Innsbruck, Anichstraße 35, 6020 Innsbruck, Austria

Full list of author information is available at the end of the article
}

\section{Background}

Osteoarthritis (OA) of the temporomandibular joint (TMJ) is a degenerative process characterized by deterioration of articular tissue with concomitant radiographically detectable osseous changes including flattening, sclerosis, osteophytes, and erosion involving the condyle and/or articular eminence [1-3]. The prevalence of TMJ $\mathrm{OA}$ is estimated to range from 8 to $35 \%$ in the general 
population based on radiographic assessment $[4,5]$. The clinical diagnosis of TMJ OA depends on clinical features such as joint pain and crepitus noises [6,7]. Patients may experience prolonged pain and disability which causes chronic symptoms becoming more refractory to traditional medical treatment approaches $[8,9]$.

It is now thought that the underlying mechanisms contributing significantly to the initiation and development of OA are synovial inflammation and increased subchondral bone turnover [10,11]. Increased bone remodeling is thought to characterize OA progression, with early bone loss accompanied by remodeling, subchondral bone sclerosis and full cartilage loss [12].

More recently, erosive alterations in TMJ OA has become a focus of interest. The pathophysiology of erosive alterations is unclear and the question whether erosive OA is as a separate entity or a more severe form of TMJ OA remains unresolved. Condylar erosion (CE) of the TMJ involves articular cartilage and adjacent cortical and subcortical bone structures [13, 14]. CE is understood as an inflammatory subset of OA, considered as a sign of progressive OA [14, 15], and has been linked to characteristic clinical findings, including irregular or deviating jaw function, joint sounds, and pain $[15,16]$. Further, $\mathrm{CE}$ is associated with magnetic resonance imaging (MRI) findings of disk displacement $[17,18]$ and should be adequately addressed in terms of diagnostic and therapeutic management to prevent changes in dentofacial morphology or limited mandibular growth [19].

For the evaluation of structural TMJ OA characteristics, computed tomography $[20,21]$ and cone beam computed tomography (CBCT) [22, 23] are currently considered as the gold standard. OA, however, is currently recognized as an entire joint failure [24], i.e., MRI allowing multiplanar visualization of all the joint components has been increasingly used in TMJ OA and inflammatory joint diseases as an outcome measure $[25,26]$. Effusion and bone marrow lesions are acknowledged as important markers for symptomatology and prognosis in both TMJ rheumatoid arthritis and OA.

To the best of the authors' knowledge, there are no studies available addressing the role of clinical and imaging parameters in the definition of TMJ CE in a multivariate design. Thus, the aim of the present study was to identify the most associated variables for prediction of TMJ CE in patients with TMD pain according to clinical parameters and magnetic MRI findings.

\section{Methods}

Study design, population, inclusion and exclusion criteria The study group, selected over a period of approximately 20 years, consisted of 124 consecutive patients with a unilateral TMJ pain condition of arthralgia. There were 222 females and 26 males, aged between 18 and 67 years with a mean age of 37.5 years. All participants had initially been referred to, and were examined at the Orofacial Pain Unit, Department of Oral and Maxillofacial Surgery, Medical University of Innsbruck.

The subjects were informed about the study procedure, and informed consent was received from each participant. This retrospective study followed the medical protocols and ethics outlined in the Declaration of Helsinki. Given the retrospective nature of this study, ethical approval of the study was waived by the Ethics Committee of the Medical University of Innsbruck.

Clinical data, consisting of demographic, clinical and treatment records were collected from chart records. The clinical assessment was performed by one clinician (RE) specialized in temporomandibular disorders (TMD) and orofacial pain. Each subject completed a visual pain rating to assess severity of pain by using a $100-\mathrm{mm}$ visual analogue scale, ranging from 0 (no pain) to 100 (very severe pain), i.e. patients registered the mean pain perceived in the last seven days. This scale has been used extensively in randomized trials and has shown good construct validity in comparison with other pain measures [27, 28]. Indications for the MRI of each subject at baseline were retrieved from clinical records.

The inclusion consisted of (i) a unilateral Research Diagnostic Critera (RDC) for TMD diagnosis of unilateral arthralgia, (ii) a pain duration of at least 1 month, (iii) a pain intensity of at least $10 \mathrm{~mm}$ as assessed by a VAS, (iv) an age between 18-70 years, (v) in cases of tooth loss, a posterior tooth missing for more than 6 months, and (vi) MRI performed within 2 weeks after the clinical examination. The exclusion criteria included (i) a RDC/ TMD diagnois of myofacial pain with limited mouth opening, (ii) a RDC/TMD diagnosis of disk displacement with reduction, (iii) a cervical or head pain, (iv) an acute head or neck infection, (v) a diagnosis of a collagen vascular disease, (vi) a previous head or neck trauma in history, (vii) a history of previous TMD treatment, and (viii) a diagnosis of a debilitating mental or physical illness.

\section{MRI data acquisition}

MRI was carried out with a $1.5 \mathrm{~T}$ MR scanner (Vision, Siemens AG, Erlangen, Germany) and a dedicated circular-polarized transmit-and-receive TMJ coil. The data were collected on a $252 \times 256$ matrix with a field of view of $145 \mathrm{~mm}$ giving a pixel size of $0.60 \times 0.57 \mathrm{~mm}$. With the patient in a supine position, 15 paracoronal and 8 para-sagittal slices were obtained of each TMJ using a TSE (turbo spin echo)-PD (proton density) sequence (repitition time of $2800 \mathrm{~ms}$, echo time of $15 \mathrm{~ms}$ ) and a TIRM (turbo inversion recovery magnitude) sequence 
(repitition time of $4000 \mathrm{~ms}$, echo time of $30 \mathrm{~ms}$, inversion time of $150 \mathrm{~ms}$ ) with thin slices of $3 \mathrm{~mm}$. MR images were corrected to the horizontal angulation of the long axis of the condyle [29].

Each subject received an individual nonferromagnetic intermaxillary device to obtain the different mouth opening positions. Sequential bilateral T1- and T2- weighted images were made at the closed mouth and the respective maximum mouth opening positions. Those T1-weighted images were selected for analysis of the disk-condyle relationship that depicted the disk, condyle, articular eminence, and glenoid fossa. Normal disk position was defined by location of the posterior band of the disk at the superior or 12 o'clock position relative to the condyle, whereas disk displacement was defined as the posterior band of the disk being in an anterior, anteromedial, anterolateral, medial, or lateral position relative to the superior part of the condyle. Diagnosis of TMJ disk-condyle relationship was categorized as normal and disk displacement with and without reduction, and defined according to the finding of a closed mouth-related diagnosis of absence or presence of disk displacement associated with or without an open mouth-related interposition of the disk between the condyle and the articular eminence [30]. MRI diagnosis of condylar osteoarthrosis was defined by the presence of subchondral sclerosis, erosion and osteophyte [29,31]. MRI diagnosis of CE was defined as an interruption or absence of the cortical lining of the condyle [32,33]. On the T2-weighted images, joint effusion was identified as an area of high signal intensity in the region of the joint space. When more than a line of high signal was evident in at least 2 consecutive sections, it was considered positive for TMJ effusion [34]. Bone marrow edema was defined by the presence of a hypointense signal on T1-weighted images and a hyperintense signal on T2-weighted images (Figs. 1 and 2) [35].

Reliability scores were determined by administering the imaging criteria on a set group of images, thereby allowing for intrarater comparison. The intraobserver reliability was strong $($ Kappa $>0.85)$ to exellent $($ Kappa $=1.00)$ for all of the MRI diagnoses.

\section{Data analysis}

Sample size was established at 239 TMJs based on the following assumptions: the prevalence of CE among TMJ arthralgia patients would be $40 \%$, and $15 \%$ among asymptomatic controls [36, 37], with an alpha error of 0.05 , and a statistical power of $95 \%$. For sample size estimation, the G*Power software (version 3.1) was applied.

Categorical variables were analysed by the chisquare test, and continuous variables were expressed as mean \pm SD with an independent-samples $t$ test. Stepwise logistic regression analyses were performed to identify

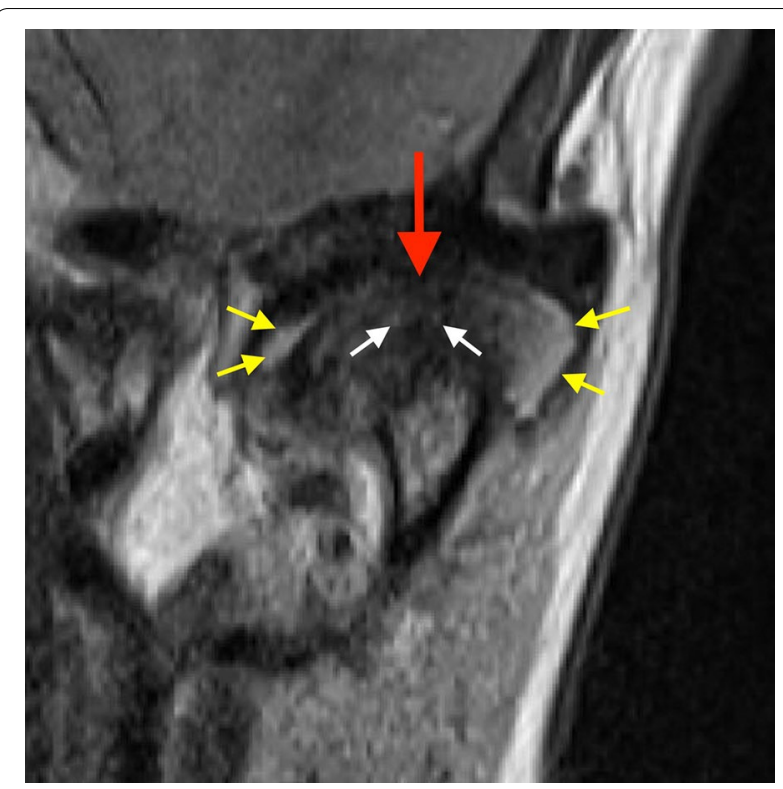

Fig. 1 Closed-mouth-related MR images in a 61-year-old female with a 18-month history of left TMJ pain, a TMJ pain-side-related clinical diagnosis of TMJ AR, and the MRI finding of disk displacement without reduction, $\mathrm{CE}$, bone marrow edema, and effusion. Coronal MR image shows condyle with CE (red arrow), bone marrow edema (white arrows), and effusion in the superior joint compartment (yellow errors)

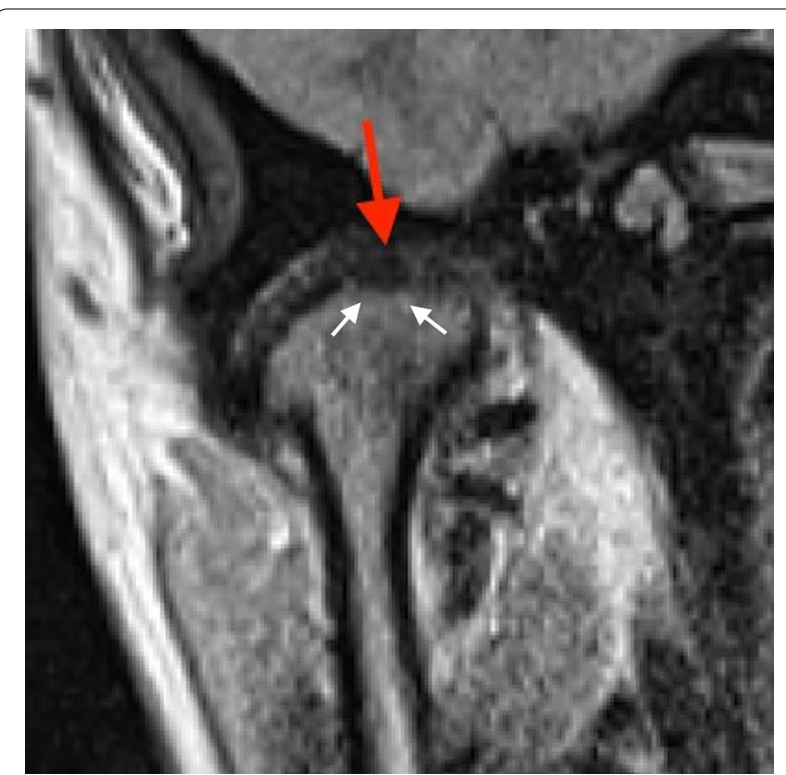

Fig. 2 Closed-mouth-related MR images in a 18-year-old female with a 12-month- history of right TMJ pain, a TMJ pain-side-related clinical diagnosis of TMJ'AR and disk displacement without reduction with limited opening (DDwoR/wLO)', and the MRI finding of disk displacement without reduction and CE. Coronal MR image shows condyle with CE (red arrow) and bone marrow edema (white error) 
multivariate predictors of CE. Based on previous studies, the variables, which were included in the model, were age (years), gender, time since pain onset, pain intensity, the number of missing posterior teeth, bone marrow edema, and effusion [14, 38]. Significance was set at $P<0.05$. For the statistical analysis, the PASW 27.0 (SPSS Statistics, IBM, Chicago) package was used.

A receiver operating characteristic (ROC) curve was used to describe the discrimination abilities of the predictive indicators. The area under the curve (AUC) provides a global summary statistic of test accuracy, and guidelines suggest that $0.5<\mathrm{AUC} \leq 0.7$ represent low accuracy, $0.7<$ AUC $\leq 0.9$ moderate accuracy, and $0.9<$ AUC $\leq 1.0$ represents high accuracy. An AUC above 0.75 is considered as good. The $95 \%$ confidence interval (CI) was calculated, and $P<0.05$ was considered to indicate statistical significance. For the statistical analysis, the PASW 27.0 (SPSS Statistics, IBM, Chicago) package was used.

\section{Results}

At least 1 TMJ with CE was present in $61.3 \%$ of the 124 patients with TMJ arthralgia. CE was found in $50.0 \%$ of the TMJs with arthralgia, and in $25.8 \%$ of TMJs without pain. Analysis of side-related data showed CE $(50.0 \%$ vs $25.8 \%)(P<0.001)$ to be more prevalent in TMJs with arthralgia than in those without pain (Table 1).

The clinical and MRI indicators assessed in this study are listed in Table 2. Nine indicators were significantly different between the $\mathrm{CE}$ and non-CE groups: missing posterior teeth $(P<0.001)$, pain intensity $(P=0.002)$, RDC/TMD of arthralgia $(P=0.004)$, RDC/TMD of arthralgia coexistant with DDwoR/wLO $(P<0.001)$, and MRI findings of disk displacement with reduction $(P=0.001)$, disk displacement without reduction $(P=0.001)$, subchondral sclerosis $(P=0.001)$, bone marrow edema $(P<0.001)$, and effusion $(P=0.001)$.

Using binary multivariate logistic analysis, 4 risk factors were identified that correlated best as predictors of $\mathrm{CE}$ : missing posterior teeth (odds ratio $[\mathrm{OR}]=1.42$;
$P=0.018), \quad$ RDC/TMD of arthralgia coexistant with DDwoR/wLO (OR=3.30, $P=0.007)$, MRI finding of disk displacement without reduction $(\mathrm{OR}=10.96$, $P<0.001$ ), and MRI finding of bone marrow edema (OR 11.97, $P<0.001$ ). Based on the modeling study, the probability of $\mathrm{CE}$ was calculated according to the following equation: $P(\mathrm{CE})=1 /(1+\exp (\mathrm{ODDS}))$, where ODDS $=-3.129+0.352$ missing posterior teeth +0.686 $\mathrm{DDwoR} / \mathrm{wLO}+2.234$ disk displacement without reduction +2.482 bone marrow edema (Table 3 ).

ROC curves of each independent variable, and the multivariate logistic model are plotted in Fig. 3. The AUC demonstrated statistical significance and a high diagnostic value for the logistic model $(\mathrm{AUC}=0.916, \mathrm{SE}=0.018$, $P<0.001,95 \%$ CI: 0.885 to 0.954 ). There was an overall prediction accuracy of $83.9 \%$ using multivariate logistic function when classification was determined using all data points (Table 4). The rates of correct prediction were 81.4\% for the CE cases (sensitivity) and $85.2 \%$ for the controls (specificity), and the positive and negative predictive values were 74.5 and $89.6 \%$, respectively.

\section{Discussion}

The results of MRI in the present study showed TMJ arthralgia patients to be associated with a high rate of CE (61.3\%). These findings compare favourably with the observations of other studies reporting TMJ CE to occur in TMJ pain patients with frequencies ranging from 34 to $85 \%[15,39,40]$, whereas in asymptomatic subjects rates are described to range from $0 \%$ to $7.4 \%[15,37,39]$. Concerning the observed prevalence rates of CE (50\%) in TMJs with arthralgia, the findings may correspond to those of previous research reports describing prevalences CBCT findings of CE in TMJ arthralgia with $60 \%$ [16], and in TMJ arthritis with 94\% [2]; the frequencies in asymptomatic TMJs being described with ranges from $6 \%$ [30] to $21 \%$ [16]. However, the results may be not directly comparable as in these studies confounding variables were not considered in a multivariate design, i.e.

Table 1 Condylar erosion by subject and TMJ side demonstrated by MRI

\begin{tabular}{|c|c|c|c|c|}
\hline \multirow[t]{2}{*}{ Distribution of CE } & \multirow[t]{2}{*}{ Subjects $(n=124)$} & \multicolumn{3}{|l|}{ TMJs } \\
\hline & & $\begin{array}{l}\text { TMJs with arthralgia } \\
(n=124)\end{array}$ & $\begin{array}{l}\text { TMJs without pain } \\
(n=124)\end{array}$ & Total $(n=248)$ \\
\hline \multicolumn{5}{|l|}{ Presence of CE } \\
\hline TMJ arthralgia side only (n) (\%) & $44(35.5)$ & $44(71.0)$ & - & $44(17.7)$ \\
\hline TMJ non-pain side only (n) (\%) & $14(11.3)$ & - & $14(43.8)$ & $14(5.7)$ \\
\hline TMJ arthralgia and non-pain side (n) (\%) & $18(14.5)$ & $18(29.0)$ & $18(56.3)$ & $36(14.5)$ \\
\hline Total (n) (\%) & $76(61.3)$ & $62(50.0)$ & $32(25.8)$ & $94(37.9)^{\mathrm{a}}$ \\
\hline
\end{tabular}

CE condylar erosion, TMJ temporomandibular joint, MRI magnetic resonance imaging, $n$ number of TMJs, (\%) percent

a $P<0.0001$, based on chi-squared test 
Table 2 Clinical and MRI indicators to predict condylar erosion of the TMJ

\begin{tabular}{|c|c|c|c|c|}
\hline Predictors & $\begin{array}{l}\text { TMJs with condylar } \\
\text { erosion }(n=94)\end{array}$ & $\begin{array}{l}\text { TMJs without condylar } \\
\text { erosion }(n=154)\end{array}$ & Total $(n=248)$ & $P$ Value \\
\hline \multicolumn{5}{|l|}{ Clinical items } \\
\hline Age (years) (mean $\pm S D)$ & $39.0(14.4)$ & $36.6(12.9)$ & $37.5(13.5)^{\mathrm{a}}$ & 0.174 \\
\hline Gender (n) (\% female) & $88(93.6)$ & $134(87.0)$ & $222(89.5)^{b}$ & 0.073 \\
\hline Missing posterior teeth (mean) & 1.56 & 0.78 & $0.124^{\mathrm{a}}$ & $<0.001$ \\
\hline Time since pain onset (weeks) (mean \pm SD) & $13.9(26.0)$ & $10.8(27.1)$ & $12.0(26.7)^{\mathrm{a}}$ & 0.377 \\
\hline Pain intensity $(\mathrm{mm})($ mean \pm SD) & $32.3(32.3)$ & $20.2(28.7)$ & $24.8(30.6)^{\mathrm{a}}$ & 0.002 \\
\hline \multicolumn{5}{|l|}{ TMD/RDC diagnosis } \\
\hline Arthralgia (n) (\%) & $24(25.5)$ & $44(28.6)$ & $66(26.6)^{b}$ & 0.004 \\
\hline Arthralgia with DDwoR/wLO (n) (\%) & $38(40.4)$ & $18(11.7)$ & $56(22.6)^{b}$ & $<0.001$ \\
\hline \multicolumn{5}{|l|}{ MRI items } \\
\hline Disk displacement with reduction (n) (\%) & $20(21.3)$ & $64(41.6)$ & $48(33.9)^{b}$ & 0.001 \\
\hline Disk displacement without reduction (n) (\%) & $70(74.5)$ & $25(16.2)$ & $95(38.3)^{b}$ & 0.001 \\
\hline Subchondral sclerosis (n) (\%) & $38(40.4)$ & $96(62.3)$ & $134(54.0)^{\mathrm{b}}$ & 0.001 \\
\hline Osteophyte (n) (\%) & $30(31.9)$ & $33(21.4)$ & $63(25.4)^{b}$ & 0.066 \\
\hline Bone marrow edema $(n)(\%)$ & $61(69.4)$ & $20(13.0)$ & $48(32.7)^{b}$ & $<0.001$ \\
\hline Effusion (n) (\%) & $28(29.8)$ & $19(12.3)$ & $47(19.0)^{b}$ & 0.001 \\
\hline
\end{tabular}

$T M J$ temporomandibular joint, $M R I$ magnetic resonance imaging, $S D$ standard deviation, (\%) percent, $n$ number of TMJs

a Based on independent samples $t$ test

${ }^{\mathrm{b}}$ Based on chi-squared test

Table 3 Results of binary multivariate logistic regression analysis of influencing factors of condylar erosion of the TMJ

\begin{tabular}{|c|c|c|c|c|c|c|}
\hline Predictors & B & S.E & Wald statistic & $P$ & Odds ratio & $95 \% \mathrm{Cl}$ \\
\hline \multicolumn{7}{|l|}{ Clinical items } \\
\hline Missing posterior teeth (mean \pm SD) & 0.352 & 0.149 & 5.574 & 0.018 & 1.422 & $1.062-1.906$ \\
\hline \multicolumn{7}{|l|}{ TMD/RDC diagnosis } \\
\hline Arthralgia with DDwoR/wLO (n) (\%) & 0.686 & 0.445 & 7.198 & 0.007 & 3.300 & 1.379-7.894 \\
\hline \multicolumn{7}{|l|}{ MRI items } \\
\hline Disk displacement without reduction (n) (\%) & 2.234 & 0.392 & 37.414 & $<0.001$ & 10.959 & $5.087-23.607$ \\
\hline Bone marrow edema (n) (\%) & 2.482 & 0.406 & 33.876 & $<0.001$ & 11.968 & $5.400-26.488$ \\
\hline
\end{tabular}

TMJ temporomandibular joint, MRI magnetic resonance imaging, SD standard deviation, (\%) percent, $n$ number of TMJs

studies failed to take into account relevant clinical and MRI parameters simultaneously.

To the best of our knowledge, this is the first study to provide relative odds for the estimation of CE of the TMJ in a multivariate design using logistic regression techniques for analysis. It provides a perspective to the contribution of clinical and MRI parameters to the occurrence of a CE. While the clinical parameters of missing posterior teeth (1: 1.42), and RDC/TMD of arthralgia coexistant with DDwoR/wOL (1: 3.30) contributed a minor to moderate amount to the change in risk, a clear definition of the CE group was evident for the MRI variables of disk displacement without reduction (1: 10.96), and bone marrow edema (1: 11.97). Therefore, based on this study, the presence of MRI findings of disk displacement without reduction and bone marrow edema may be considered a dominant factor in the definition of $\mathrm{CE}$ of the TMJ. Considering the aspect of arthritic TMJ conditions as an underlying mechanism in the development of changes in dentofacial morphology or limited mandibular growth [41, 42], further investigations are indicated to clarify which additional clinical and/or MRI variables may be associated with an elevated risk for arthritic CE signs of TMJ OA, while only a prospective cohort study rather than a case-control study will estimate the etiologic contribution of defined variables to $C E$.

Most studies have focused on the identification of risk factors of clinical TMD diagnoses [34, 38], while an effective risk prediction tool for $C E$ is lacking. In the current research, CE was linked to 9 factors screened by 


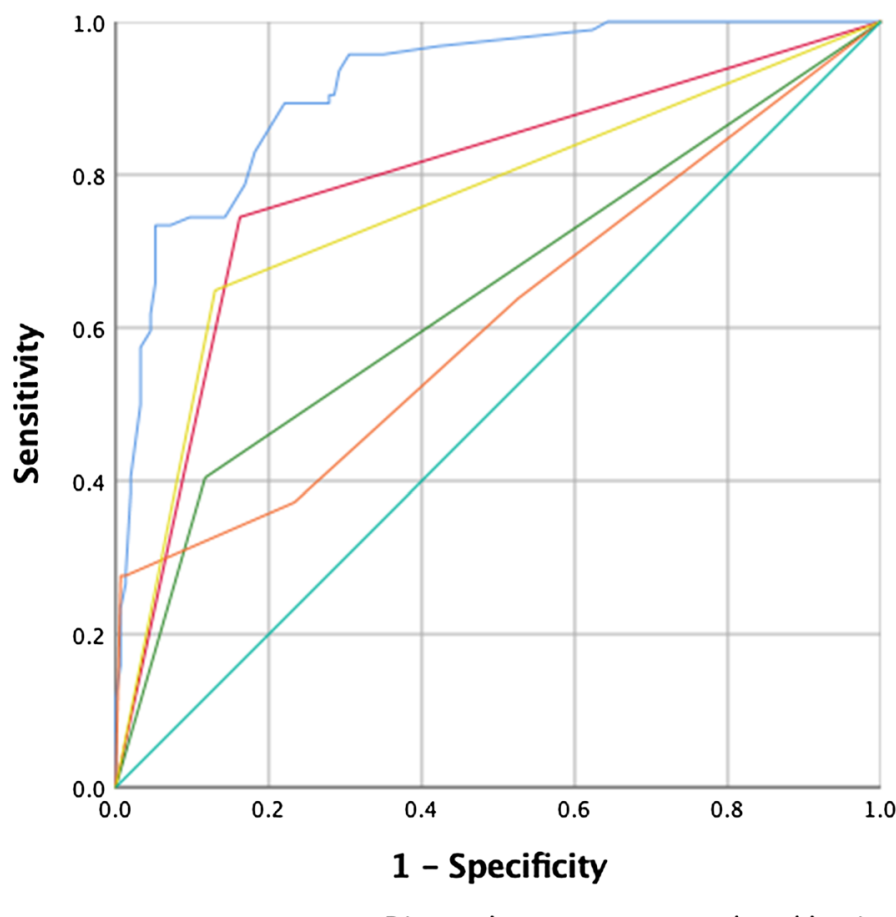

Logistic regression model Disk displacement without reduction

- DDwoR/wLO

Missing posterior teeth

- Bone marrow edema

- Reference Line

Diagonal segments are produced by ties.

Fig. 3 Receiver operating characteristic (ROC) curves of multivariate logistic regression model illustrating the predictive capacity of the model. The area under the curve of ROC curves of individual variables, namely MRI finding of disk displacement without reduction, RDC/TMD of arthralgia coexistant with disk displacement without reduction with limited mouth opening (DDwoR/wOL), missing posterior teeth, and bone marrow edema

Table 4 Percentages of correct classifications of TMJs conducted by binary multivariate logistic regression analysis

\begin{tabular}{llll}
\hline & Predicted group membership & & \\
\hline Actual classification & Non-condylar erosion (\%) & Condylar erosion (\%) & Total \\
Non-condylar erosion & $138(87.0)$ & $16(13.0)$ & 154 \\
Condylar erosion & $24(23.9)$ & $70(76.6)$ & 94 \\
Total & 156 & 92 & 248 \\
\hline
\end{tabular}

TMJ temporomandibular joint, (\%) percent, $83.9 \%$ of original grouped cases correctly classified

multivariate logistic regression analysis, but only 4 factors used as predictors were included in the regression analysis. Selected predictors, i.e., missing posterior teeth, RDC/TMD of arthralgia coexisting with DDwoR/wLO, and MRI findings of disk displacement without reduction and bone marrow edema were significantly correlated with $\mathrm{CE}$, a finding which is consistent with previous research [43-46].

In classifying $83.9 \%$ of participants, with an AUC of 0.916 , the prediction model developed by multivariate regression analysis was efficient. In order to classify the high-risk population with active condylar alterations in the first clinical environment, the prediction model can be used as a risk prediction method for $\mathrm{CE}$, and thereby provide valuable information for further clinical followup and treatment approaches.

If patients are identified that they are at a high risk by this prediction, clinicians may be able to control some important risk factors to reduce the risk of active progressive condylar alterations. In practice, considering a hypothetical case, involving a patient with TMJ arthralgia, where the number of missing posterior teeth $=4$, $\mathrm{DDwoR} / \mathrm{wLO}=0$, disk displacement without reduction $=1$, and bone marrow edema each $=1$, the odds prediction equation is ODDS $=\exp (-3.129+0.352 \times 4+0$ $.686 \times 0+2.234 \times 1+2.482 \times 1)$, and the $P(C E)=0.952$. That is, the model predicts that $95 \%$ of the TMJs may be at risk for the occurrence of $\mathrm{CE}$. 
Several factors are acknowledged as risk items for the development of CE [14, 47, 48]. They comprise gender, age systemic, hormonal factors, and arthritis [49, 50]. Mechanical factors include disk displacement, occlusion, trauma, and increased friction at the joint $[51,52]$. Further, overloading is the main unterlying disorder in any synovial joint, including the TMJ [53], and may contribute to the initiation of various phenomena, such as increased friction, adhesive forces, and shear stress [54, 55]. A compromised lubrication generates various levels of friction between the articular surfaces. The production of non-reducing disk phenomena and degenerative articular surface alterations that may also cause the onset of bone marrow edema and CE may be triggered by longterm mild friction [56-59].

The preventive use of MRI to identify subtypes of TMJ ID and OA may be suggested by this research [60, 61]. Early MRI diagnosis of disk displacement without reduction, bone marrow edema, and potentially associated erosive condylar processes can become an important factor in prevention and early treatment as it may prevent changes in dentofacial morphology or restricted mandibular development $[41,42]$. In view of the fact that etiology, prognostic aspects, and treatment implications are the main aspects for the utility of diagnostic classifications [60], ongoing research is necessary to determine how well specific MRI findings of ID, OA and inflammatory TMJ alterations may demonstrate differences in pathogenesis, treatment, and prognosis.

Prognostic prediction models guide physicians upon therapeutic management and have become a standard to aid clinical decision-making. Demographics, clinical, and imaging characteristics, and specific test results are applied to derive these models, thereby estimating the probability of developing a particular outcome [62, 63]. In the development phase, multivariable regression techniques are used in model development [64]. In the validation phase, patient data not included in the development process are applied to test the developed model. With regard to the prognostic prediction model developed in the present study, it is therefore advised that external validation is performed in ongoing studies before considering to incorporate this model into clinical practice.

The present study may have failed to adequately assess the involved chronic TMJ pain patients. Chronic pain is considered a multidimensional emotional and sensory experience comprising affective, sensorial, and cognitiveevaluative aspects $[65,66]$. The current study was limited by addressing only the sensory discriminative dimension of pain, i.e. further studies may have to apply multidimensional instruments to assess the chronic pain condition $[6,66]$. Another limitation concerns the aspect that in studies assessing differences between study groups, the ideal control group should be the least symptomatic and the study sample should be the most diseased available. The TMJ arthralgia group may have contained too much "noise" by including TMJs sides with signs of associated palpation pain of the ipsilateral masticatory muscles, i.e., in some cases myalgia may have mimicked TMJ arthralgia [67]. Ongoing studies that involve a wellness "TMJ arthralgia" group characterized by the absence of associated signs of muscle palpation pain should be encouraged.

Observer bias from the radiologist may tend to have a significant impact on the assessment of MRI variables. Various factors such as quality of an image, specific criteria for interpretation, and training can affect observer performance. The current study used MRI images of high quality and applied well-defined criteria in the assessment of MRI variables, while intraobserver reliability was strong for the diagnostic criteria applied. However, study designs of medical imaging should routinely include more than one observer [68], i.e., consequently, overestimation of the significance of some MRI items to the described TMJ arthralgia groups may have occurred.

\section{Conclusion}

The established prediction model using the risk factors of TMJ arthralgia may be useful for predicting the risk of CE. The data suggest MRI indicators as dominant factors in the definition of CE. Further research is needed to improve the model, and confirm the validity and reliability of the model.

\section{Abbreviations \\ AUC: Area under the curve; CE: Condylar erosion; DDwoR/wLO: Disk displace- ment without reduction with limited opening; MRI: Magnetic resonance imag- ing; OA: Osteoarthritis; OR: Odds ratio; RDC/TMD: Research diagnostic criteria for temporomandibular disorders; TMJ: Temporomandibular joint.}

\section{Acknowledgements}

Not applicable.

\section{Authors' contributions}

A.B. and A.R. collected the data, contributed to drafting the article; R.E. developed the design, analyzed the data, contributed to drafting and critical revision of the article; L.H. contributed to critical revision of the article. The authors read and approved the final manuscript.

\section{Funding}

This research did not receive any specific grant from funding agencies in the public, commercial or not-for-profit sectors.

\section{Availability of data and materials}

All data generated or analyzed during this study are not publicly available due to ethical and confidentiality reasons. The data will only be shared in aggregate form as presented in the figures and tables. 


\section{Declarations}

\section{Ethics approval and consent to participate}

The study followed the Declaration of Helsinki on medical protocol and ethics. Given the retrospective nature of this study, ethical approval of the study was waived by the Ethics Committee of the Medical University of Innsbruck. Informed consent was obtained from each participant included in the study.

\section{Consent for publication}

Not applicable.

\section{Competing interests}

The authors declare that they have no competing interests.

\section{Author details}

'Orofacial Pain and TMD Unit, University Clinic of Oral and Maxillofacial Surgery, Medical University of Innsbruck, Anichstraße 35, 6020 Innsbruck, Austria. ${ }^{2}$ Otto Von Guericke University of Magdeburg, Magdeburg, Germany. ${ }^{3}$ University Clinic of Oral and Maxillofacial Surgery, Medical University of Innsbruck, Innsbruck, Austria. ${ }^{4}$ University Clinic of Radiology, Medical University of Innsbruck, Innsbruck, Austria.

Received: 19 October 2020 Accepted: 24 June 2021

Published online: 24 July 2021

\section{References}

1. Talaat W, Al Bayatti S, AI KS. CBCT analysis of bony changes associated with temporomandibular disorders. Cranio. 2016;34:88e94

2. Comert Kilic S, Kilic N, Sumbullu MA. Temporomandibular joint osteoarthritis: cone beam computed tomography findings, clinical features, and correlations. Int J Oral Maxillofac Surg. 2015;44:1268e74.

3. Koyama J, Nishiyama H, Hayashi T. Follow-up study of condylar bony changes using helical computed tomography in patients with temporomandibular disorder. Dentomaxillofac Radiol. 2007;36:472e7.

4. Brooks SL, Westesson PL, Eriksson L, Hansson LG, Barsotti JB. Prevalence of osseous changes in the temporomandibular joint of asymptomatic persons without internal derangement. Oral Surg Oral Med Oral Pathol. 1992;73:118-22

5. Kalladka M, Quek S, Heir G, Eliav E, Mupparapu M, Viswanath A. Temporomandibu- lar joint osteoarthritis: diagnosis and long- term conservative management: a topic review. J Indian Prosthodont Soc. 2014;14:6-15.

6. Dworkin SF, LeResche L. Research diagnostic criteria for temporomandibular disorders: review, criteria, examinations and specifications, critique. J Craniomandib Disord. 1992;6:301-55.

7. Schiffman E, Ohrbach R, Truelove E, Look J, Anderson G, Goulet JP, et al. Diagnostic criteria for temporomandibular disorders (DC/TMD) for clinical and research applications: recommendations of the international RDC/ TMD consortium network and orofacial pain special interest group. J Oral Facial Pain Headache. 2014;28:6-27.

8. Dworkin SF, Huggins KH, LeResche L, Von Korff M, Howard J, Truelove E, et al. Epidemiology of signs and symptoms in temporomandibular disorders: clinical signs in cases and controls. JADA. 1990;120:273-81.

9. Dworkin $\mathrm{RH}$. Which individuals with acute pain are most likely to develop a chronic pain syndrome? Pain Forum. 1997;6:127-36.

10. Weber A, Chan PMB, Wen C. Do immune cells lead the way in subchondral bone disturbance in osteoarthritis? Prog Biophys Mol Biol. 2019;148:21-31

11. Wang XD, Zhang JN, Gan YH, Zhou YH. Current understanding of pathogenesis and treatment of TMJ osteoarthritis. J Dent Res. 2015:94:666-73.

12. Burr DB, Gallant MA. Bone remodelling in osteoarthritis. Nat Rev Rheumatol. 2012;8:665-73.

13. Sokoloff L. Pathology and pathogenesis of osteoarthrosis. In: McCarty DJ, Hollander $\mathrm{J}$, editors. Arthritis and allied conditions: a textbook of rheumatology. Lea \& Febiger; 1979. p. 1135-1153.

14. Emshoff R. Pathophysiology of intracapsular inflammation and degeneration. In: Greene CS, Laskin DM, editors. Treatment of temporomandibular disorders: bridging the gap between advances in research and clinical patient management. Quintessence Publishing; 2013. pp. 33-46.
15. Cevidanes LH, Hajati AK, Paniagua B, Lim PF, Walker DG, Palconet G, et al. Quantification of condylar resorption in temporomandibular joint osteoarthritis. Oral Surg Oral Med Oral Pathol Oral Radiol Endod. 2010;110:110-7.

16. Emshoff R, Bertram F, Schnabl D, Stigler R, Steinmaß। O, Rudisch A. Condylar erosion in patients with chronic temporomandibular joint arthralgia: a cone-beam computed tomography study. J Oral Maxillofac Surg. 2016;74:1343e1-8.

17. Campos MI, Campos PS, Cangussu MC, Guimarães RC, Line SR. Analysis of magnetic resonance imaging characteristics and pain in temporomandibular joints with and without degenerative changes of the condyle. Int J Oral Maxillofac Surg. 2008;37:529-34.

18. Gil C, Santos KC, Dutra ME, Kodaira SK, Oliveira JX. MRI analysis of the relationship between bone changes in the temporomandibular joint and articular disc position in symptomatic patients. Dentomaxillofac Radiol. 2012:41:367-72.

19. Silva MAG, Pantoja LLQ, Dutra-Horstmann KL, Valladares-Neto J, Wolff $\mathrm{FL}$, Porporatti AL, et al. Prevalence of degenerative disease in temporomandibular disorder patients with disc displacement: a systematic review and meta-analysis. J Craniomaxillofac Surg. 2020:48:942-55.

20. Westesson PL, Katzberg RW, Tallents RH, Sanchez-Woodworth RE, Svensson SA. CT and MR of the temporomandibular joint: comparison with autopsy specimens. AJR Am J Roentgenol. 1987;148:1165-71.

21. Manzione JV, Katzberg RW, Brodsky GL, Seltzer SE, Mellins HZ. Internal derangements of the temporomandibular joint: diagnosis by direct sagittal computed tomography. Radiology. 1984;150:111-5.

22. Hashimoto $K$, Arai $Y$, Iwai $K$, et al. A comparison of a new limited cone beam computed tomography machine for dental use with a multidetector row helical CT machine. Oral Surg Oral Med Oral Pathol Oral Radiol Endod. 2003;95:371-7.

23. Honey OB, Scarfe WC, Hilgers MJ, Klueber K, Silveira AM, Haskell BS, et al. Accuracy of cone-beam computed tomography imaging of the temporomandibular joint: Comparisons with panoramic radiology and linear tomography. Am J Orthod Dentofacial Orthop. 2007;132:429-38.

24. Abramson SB, Attur M. Developments in the scientific understanding of osteoarthritis. Arthritis Res Ther. 2009:11:227.

25. De Riu G, Vaira LA, Carta E, Meloni SM, Sembronio S, Robiony M. Bone marrow nucleated cell concentrate autograft in temporomandibular joint degenerative disorders: 1-year results of a randomized clinical trial. J Craniomaxillofac Surg. 2019;47:1728-38.

26. Arabshahi B, Dewitt EM, Cahill AM, Kaye RD, Baskin KM, Towbin $\mathrm{RB}$, et al. Utility of corticosteroid injection for temporomandibular arthritis in children with juvenile idiopathic arthritis. Arthritis Rheum. 2005;52:3563-9.

27. Price DD, McGrath PA, Rafii A. The validation of visual analogue scales as ratio scale measures for chronic and experimental pain. Pain 1983;17:45-56.

28. Minakuchi H, Kuboki T, Matsuka Y, Maekawa K, Yatani H, Yamashita A. Randomized controlled evaluation of non-surgical treatments for temporomandibular joint anterior disk displacement without reduction. J Dent Res. 2001:80:924-8.

29. Bertram S, Rudisch A, Innerhofer K, Pümpel E, Grubwieser G, Emshoff R. Magnetic resonance imaging to diagnose temporomandibualar joint internal derangement and osteoarthrosis. J Am Dent Assoc. 2001;66:75-7.

30. Tasaki MM, Westesson P-L, Isberg AM, Ren Y-F, Tallents RH. Classification and prevalence of temporomandibular joint disc displacement in patients and asymptomatic volunteers. Am J Orthod Dentofacial Orthop. 1996;109:249-62.

31. De Leeuw R, Boering G, Stegenga B, de Bont LGM. Radiographic assessment of joint osteoarthrosis and internal derangement 30 years after nonsurgical treatment. Oral Surg Oral Med Oral Pathol Radiol Endod. 1995;79:382-92.

32. Alexiou K, Stamatakis $H$, Tsiklakis K. Evaluation of the severity of temporomandibular joint osteoarthritic changes related to age using cone beam computed tomography. Dentomaxillofac Radiol. 2009:38:141-7.

33. Ahmad M, Hollender $L$, Anderson Q, Kartha K, Ohrbach R, Truelove EL, et al. Research diagnostic criteria for temporomandibular disorders (RDC/ TMD): development of image analysis criteria and examiner reliability for image analysis. Oral Surg Oral Med Oral Pathol Oral Radiol Endod. 2009:107:844-60. 
34. Emshoff R, Brandlmaier I, Gerhard S, Strobl H, Bertram S, Rudisch A. Magnetic resonance imaging predictors of temporomandibular joint pain. J Am Dent Assoc. 2003;134:705-14.

35. Larheim TA, Westesson P-L, Hicks DG, Eriksson L, Brown DA. Osteonecrosis of the temporomandibular joint: correlation of magnetic resonance imaging and histology. J Oral Maxillofac Surg. 1999;57:888-98.

36. Cevidanes LH, Walker D, Schilling J, Sugai J, Giannobile W, Paniagua B, et al. 3D osteoarthritic changes in TMJ condylar morphology correlates with specific systemic and local biomarkers of disease. Osteoarthritis Cartilage. 2014;2:1657-67.

37. Wang ZH, Jiang L, Zhao YP, Ma XC. Investigation on radiographic signs of osteoarthrosis in temporomandibular joint with cone beam computed tomography in adolescents. Beijing Da Xue Xue Bao. 2013;45:280-5.

38. Wang MQ, Xue F, He JJ, Chen JH, Chen CS, Raustia A. Missing posterior teeth and risk of temporomandibular disorders. J Dent Res. 2009;88:942-5.

39. Zhao YP, Zhang ZY, Wu YT, Zhang WL, Ma XC. Investigation of the clinical and radiographic features of osteoarthrosis of the temporomandibular joints in adolescents and young adults. Oral Surg Oral Med Oral Pathol Oral Radiol Endod. 2011;111:e27-34.

40. Imanimoghaddam M, Madani AS, Talebzadeh MR, Bagherpour A, Alimohammadi. The relationship between osseous changes of the temporomandibular joint and RDC/TMD groups in CBCT images. J Dent Mater Tech 2014;3:151-7.

41. Bertram S, Moriggls A, Rudish A, Emshoff R. Structural characteristics of bilateral temporomandibular joint disc displacement without reduction and osteoarthrosis are important determinants of horizontal mandibular and vertical ramus deficiency: a magnetic resonance imaging study. J Oral Maxillofac Surg. 2011;69:1898-904.

42. Roh HS, Kim W, Kim YK, Lee JY. Relationships between disk displacement, joint effusion, and degenerative changes of the TMJ in TMD patients based on MRI findings. J Craniomaxillofac Surg. 2012;40:283-6286.

43. Bertram F, Hupp L, Schnabl D, Rudisch A, Emshoff R. Association between missing posterior teeth and occurrence of temporomandibular joint condylar erosion: a cone beam computed tomography study. Int J Prosthodont. 2018;31:9-14.

44. Han JH, Le J, Liu MQ, Fu KY. The images of osteoarthrosis associated with anterior disc displacement without reduction detected by cone-beam CT. Zhonghua Kou Qiang Yi Xue Za Zhi. 2017;9:22-6.

45. Dias IM, Coelho PR, Assis NMSP, Leite FPP, Devito KL. Evaluation of the correlation between disc displacements and degenerative bone changes of the temporomandibular joint by means of magnetic resonance images. Int J Oral Maxillofac Surg. 2012;41:1051-7.

46. Shigeno K, Sasaki Y, Otonari-Yamamoto M, Ohata H, Shibahara T. Evaluating the mandibular condyles of patients with osteoarthritis for bone marrow abnormalities using magnetic resonance T2 mapping. Oral Radiol. 2019:35:272-9.

47. Arden N, Nevitt MC. Osteoarthritis: epidemiology. Best Pract Res Clin Rheumatol. 2006;20:3-25.

48. Milam SB. Articular disk displacements and degenerative temporomandibular joint disease. In: Sessle BJ, Bryant PS, Dionne RA, editors, Temporomandibular disorders and related pain conditions: Progress in pain research and management. IASP Press; 1995. pp. 89-112.

49. Kang SC, Lee DG, Choi JH, Kim ST, Kim YK, Ahn HJ. Association between estrogen receptor polymorphism and pain susceptibility in female temporomandibular joint osteoarthritis patients. Int J Oral Maxillofac Surg. 2007;36:391-4.

50. Wright $\mathrm{V}$, Dowson D, Longfied MD. Joint stiffness-its characterization and significance. Biomed Eng. 1969;4:8-14.
51. Asakawa-Tanne Y, Su S, Kunimatsu R, Hirose N, Mitsuyoshi T, Okamoto Y. Effects of enzymatic degradation after loading in temporomandibular joint. J Dent Res. 2015;94:337-43.

52. Moncada G, Cortés D, Millas R, Marholz C. Relationship between disk position and degenerative bone changes in temporomandibular joints of young subjects with TMD. An MRI study Clin Pediatr Dent. 2014;38:269-76.

53. Nitzan DW, Mahler Y, Simkin A. Intra-articular pressure measurements in patients with suddenly developing, severely limited mouth opening. J Oral Maxillofac Surg. 1992;50:1038-42.

54. Rahamim E, Better H, Dagan A, Nitzan DW. Electron microscope and biochemical observations of the surface active phospholipids on the articular surfaces and in the synovial fluid of the temporomandibular joint: a preliminary investigation. J Oral Maxillofac Surg. 2001;59:1326-32.

55. Zhang J, Jiao K, Zhang M, Zhou T, Liu XD, Yu SB, et al. Occlusal effects on longitudinal bone alterations of the temporomandibular joint. J Dent Res. 2013;92:253-9

56. Nitzan DW. The process of lubrication impairment and its involvement in temporomandibular joint disc displacement: a theoretical concept. J Oral Maxillofac Surg. 2001;59:36-45.

57. Tanaka E, Hirose M, Koolstra JH, van Eijden TM, Iwabuchi Y, Fujita R. Modeling of the effect of friction in the temporomandibular joint on displacement of its disc during prolonged clenching. J Oral Maxillofac Surg. 2008;6:462-8.

58. Tanaka E, Detamore MS, Tanimoto K, Kawai N. Lubrication of the temporomandibular joint. Ann Biomed Eng. 2008;36:14-29.

59. Wahaj A, Hafeez K, Zafar MS. Association of bone marrow edema with temporomandibular joint (TMJ) osteoarthritis and internal derangements. Cranio. 2017;35:4-9.

60. Sokoloff L. Pathology and pathogenesis of osteoarthrosis. In: McCarty DJ, Hollander JL, editors. Arthritis and allied conditions: A textbook of rheumatology. Lea \& Febiger; 1979. pp. 1135-53.

61. Swets JA. Measuring the accuracy of dignostic systems. Science. 1988;240:1285-93.

62. Damen JAAG, Debray TPA, Pajouheshnia R, Reitsma JB, Scholten RJPM, Moons KGM, et al. Empirical evidence of the impact of study characteristics on the performance of prediction models: a meta-epidemiological study. BMJ Open. 2019;9:e026160.

63. Collins GS, Reitsma JB, Altman DG, Moons KG. Transparent Reporting of a multivariable prediction model for Individual Prognosis or Diagnosis (TRIPOD): the TRIPOD Statement. Br J Surg. 2015;102:148-58.

64. Royston P, Moons KG, Altman DG, Vergouwe Y. Prognosis and prognostic research: developing a prognostic model. BMJ. 2009;338:b604.

65. Loeser JD, Treede RD. The Kyoto protocol of IASP basic pain terminology. Pain. 2008;137:473-7.

66. Jamison RN, Rudy TE, Penzien DB, Mosley TH Jr. Cognitive-behavioral classifications of chronic pain: replication andextension of empirically derived patient profiles. Pain. 1994;57:277-92.

67. Eliav E, Teich S, Nitzan D, El Raziq DA, Nahlieli O, Tal M. Facial arthralgia and myalgia: Can they be differentiated by trigeminal sensory assessment? Pain. 2003;104:481-90.

68. Obuchowski NA. How many observers are needed in clinical studies of medical imaging? AJR Am J Roentgenol. 2004;182:867-9.

\section{Publisher's Note}

Springer Nature remains neutral with regard to jurisdictional claims in published maps and institutional affiliations. 